\title{
Experimental Verification of the Corrected Transfer Function of a Piezoelectric Laminate Beam
}

\author{
S. O. Reza Moheimani
}

\begin{abstract}
Piezoelectric materials are finding increasing applications in active vibration control of structures. Modeling of a piezoelectric laminate, often results in an infinite-dimensional or a very high-order model. For control design purposes, such a model is simplified by removing higher frequency modes which lie out of the bandwidth of interest. Truncation can considerably perturb the in-bandwidth zeros of the truncated model. This paper suggests a method of minimizing the effect of the removed higher order modes on the low-frequency dynamics of the truncated model of a piezoelectric laminate beam by adding a zero frequency term to the low-order model of the structure. Simulations and experimental results are presented.
\end{abstract}

Index Terms-Flexible structures, model corection, model reduction, piezoelectric actuators, piezoelectric sensors.

\section{INTRODUCTION}

$\mathbf{R}$ ECENTLY, there has been an increasing interest in using piezoelectric materials as actuators and sensors for vibration control in flexible structures. Piezoelectric ceramics provide cheap, reliable, and nonintrusive means of actuation and sensing in flexible structures. When used in flexible structures, the piezoelectric ceramic films are bonded to the body of the structure using strong adhesive material. A distinct characteristic of piezoelectric actuators or sensors is that they are spatially distributed over the surface which is being sensed and/or controlled. This property makes them different from the discrete actuators and sensors which are often used in the control of flexible structures. When a piezoelectric element is stressed electrically by a voltage, its dimensions change and when it is stressed mechanically by a force, it generates an electric charge. A piezoceramic is therefore capable of acting as either a sensing or transmitting element, or both.

One of the most popular methods of modeling piezoelectric laminates, is via the modal analysis technique [1]. In this approach the solution of the the PDE, that governs the dynamics of the laminate, is assumed to consist of an infinite number of terms. Moreover, these terms are chosen to be orthogonal. This results in an infinite-dimensional model of the structure.

In control design problems, one is often interested only in designing a controller for a particular frequency range. In these situations, it is common practice to remove the modes which

Manuscript received December 21, 1998. This work was supported by the Australian Research Council and the Centre for Integrated Dynamics and Control. Recommended by Associate Editor, E. G. Collins, Jr.

The author is with the Department of Electrical and Computer Engineering and the Centre for Integrated Dynamics and Control, University of Newcastle, NSW, Australia (e-mail: reza@ee.newcastle.edu.au).

Publisher Item Identifier S 1063-6536(00)05743-2. correspond to frequencies that lie out of the bandwidth of interest and only keep the modes which directly contribute to the low-frequency dynamics of the system. This model is then used to design a controller. If such a controller is implemented on the system, say in the laboratory, the closed-loop performance of the system can be considerably different from the theoretical predictions. This is mainly due to the fact that although the poles of the truncated system are at the correct frequencies, the zeros can be far away from where they should be. Therefore, it is natural to expect that a controller designed for the truncated system may not perform well when implemented on the real system since the closed-loop performance of the system can be largely dictated by the open-loop zeros.

Reference [2] discusses the effect of out of bandwidth modes on the low-frequency zeros of the truncated model. There, it is suggested that the effect of higher frequency modes on the lowfrequency dynamics of the system can be captured by adding a zero frequency term to the truncated model to account for the compliance of the ignored modes. In this paper, we take a similar approach in the sense that we allow for a zero frequency term to capture the effect of truncated modes. However, this constant term is found such that the $\mathrm{H}_{2}$ norm of the resulting error system is minimized.

To this end, we point out that there are alternative methods for modeling of piezoelectric laminates. As an example, one can point to the recent works of Pota and Alberts in modeling of such systems using symbolic computations [3]-[5]. However, the models derived via modal analysis technique have the interesting property that they describe spatial and temporal behavior of the system. Such models can then be used in designing spatial controllers as noted in [6]-[10].

\section{Electro-Mechanical Model of a Piezoelectric LAMINATE}

Consider the piezoelectric laminate beam of Fig. 1. The beam is pinned at both ends. There are two piezoelectric patches which are used as actuators and/or sensors. Here, the subscripts " $a$," " $b$," and " $c$ " correspond to the top piezoelectric layer, the beam, and the lower piezoelectric layer. In this section we briefly explain how a transfer function can be developed for this structure. For a thorough coverage of the modeling process, the reader is referred to [11] and [12].

The " $a$ " layer serves as an actuator and the " $c$ " layer as a sensor. If a voltage $V_{a}(t, r)$ is applied to the actuating piezoelectric layer, it induces a longitudinal stress $\sigma$ given by: $\sigma(t, r)=$ $\left(E_{a} d_{31} / t_{a}\right) V_{a}(t, r)$ where $d_{31}$ is the electric charge constant of the film, $E_{a}$ is the Young's modulus of the film and $t_{a}$ is the thickness of the patch as shown in Fig. 1(b). The stress due to 


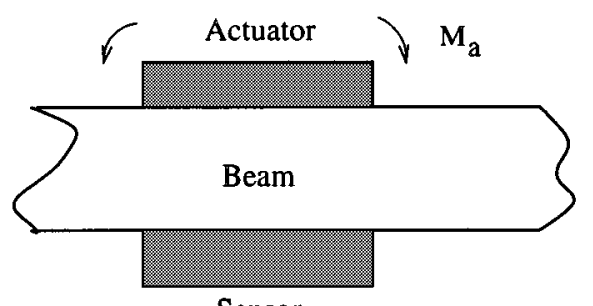

Sensor
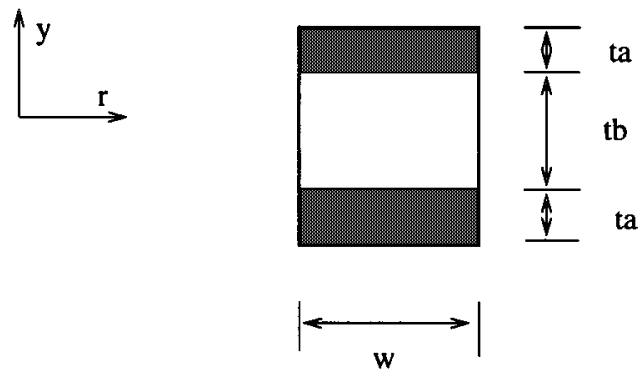

(b)

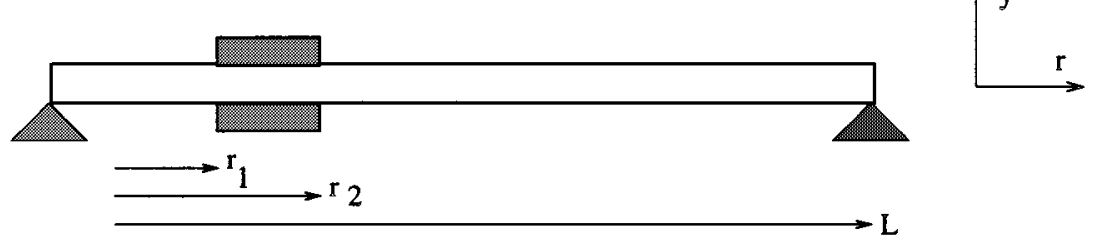

(c)

Fig. 1. The piezoelectric laminate beam.

an applied voltage produces a bending moment $M_{a}$ as shown in Fig. 1(a) along the composite system's neutral axis given by [13]: $M_{a}=\int_{t_{b} / 2}^{t_{b} / 2+t a} \sigma(t, r) w y d y=C_{a} V_{a}(t, r)$ where $C_{a}=(1 / 2) E_{a} d_{31} w\left(t_{a}+t_{b}\right)$. Obviously, $C_{a}$ is a constant which depends on the geometry of the composite system. If both layers are used as actuators with opposite polarity, then $C_{a}$ will change to $C_{a}=E_{a} d_{31} w\left(t_{a}+t_{b}\right)$.

To understand how the sensing layer works, note that when the beam is bent, it results in a strain $\epsilon(t, r)=$ $-\left(t_{b} / 2+t a\right)\left(\partial^{2} y / \partial r^{2}\right)$. Due to the piezoelectric effect, this strain produces a charge distribution per unit area $q(t, r)$ given by: $q(t, r)=\left(k_{31}^{2} / g_{31}\right) \epsilon(t, r)$ where $k_{31}$ is the piezoelectric electromagnetic coupling constant and $g_{31}$ is the piezoelectric stress constant. The total charge accumulated on the sensing layer can be found by integrating $q(t, r)$ over the entire surface area of the piezoelectric patch. That is

$$
Q(t)=\int_{r_{1}}^{r_{2}} w q(t, r) d r=-\left.w\left(\frac{t_{b}}{2}+t_{a}\right) \frac{k_{31}^{2}}{g_{31}} \frac{\partial y(t, r)}{\partial r}\right|_{r_{1}} ^{r_{2}} .
$$

Since the piezoelectric patch, once charged, can be considered as a simple parallel plate capacitor, the voltage across the two layers can be found to be

$$
V_{c}(t)=\frac{Q(t)}{C_{\mathrm{ap}}\left(r_{2}-r_{1}\right)}=\left.C_{s} \frac{\partial y(t, r)}{\partial r}\right|_{r_{1}} ^{r_{2}} .
$$

Here, $C_{\text {ap }}$ is the patch capacitance and $r_{2}-r_{1}$ is the length of the sensing layer. Moreover, $C_{s}$ is a constant which can be determined as $C_{s}=-w\left(t_{b} / 2+t_{a}\right) k_{31}^{2} / C_{\text {ap }}\left(r_{2}-r_{1}\right) g_{31}$.

The partial differential equation describing the elastic deflection of the composite beam-piezoelectric system is a Bernoulli-
Euler beam equation with an additional term due to actuating layer [3]. This equation is given as

$$
\frac{\partial^{2}}{\partial r^{2}}\left[E I \frac{\partial^{2} y(t, r)}{\partial r^{2}}-C_{a} V_{a}(t, r)\right]+\rho A \frac{\partial^{2} y(t, r)}{\partial t^{2}}=0
$$

where $E, I, A, V_{a}(t, r)$ and $\rho$ represent, respectively, the Young's modulus, moment of inertia, cross-section area, voltage across the actuating layer, and the linear mass density of the beam. Notice that if the piezoelectric patches do not cover the entire beam surface, then both $E I$ and $\rho A$ will be functions of $r$. However, since the piezoelectric layers are often thin by comparison to the base structure, we can assume that $E I$ and $\rho A$ are uniform over the length of the beam. The boundary conditions for the simply-supported beam in Fig. 1(c) are: $y(t, 0)=y(t, L)=0$ and $\left.E I\left(\partial^{2} y(t, r) / \partial r^{2}\right)\right|_{r=0}=\left.E I\left(\partial^{2} y(t, r) / \partial r^{2}\right)\right|_{r=L}=0$.

One way of solving (1) is to expand $y(t, r)$ as an infinite series in the form [14]

$$
y(t, r)=\sum_{i=1}^{\infty} q_{i}(t) \phi_{i}(r)
$$

where $\phi_{i}(r)$ are the eigenfunctions satisfying the ordinary differential equations, resulting from the substitution of (2) into (1) and its associated boundary conditions.

Following this procedure, a dynamical model of the beam is found to be

$$
\frac{\hat{V}_{c}(s)}{\hat{V}_{a}(s)}=\sum_{i=1}^{\infty} \frac{-C_{s} C_{a}\left[\phi_{i}^{\prime}\left(r_{1}\right)-\phi_{i}^{\prime}\left(r_{2}\right)\right]^{2}}{\left(s^{2}+\omega_{i}^{2}\right)}
$$


where mode shapes are given by $\phi_{i}(r)=\sqrt{2 / \rho A L} \sin (i \pi r / L)$ and resonant frequencies are $\omega_{i}=(i \pi / L)^{2} \sqrt{E I / \rho A}$. It can be observed that the transfer function (3) consists of an infinite number of resonant modes. In reality each mode is very lightly damped. The damping factor associated with each mode can be determined experimentally.

\section{CORRECTION OF THE IN-BANDWIDTh DYNAMICAL MODEL OF A LAMINATE}

The model that was developed in the previous section is for a beam with pinned-pinned boundary conditions. It is possible to carry out the same procedure for piezoelectric laminated beams with different boundary conditions. It is also possible to extend this procedure to two-dimensional structures such as piezoelectric laminated plates. In any case, the modeling of a piezoelectric laminate using the modal analysis technique results in a model that can be represented by

$$
G(s)=\sum_{i=1}^{\infty} \frac{F_{i}}{s^{2}+\omega_{i}^{2}} .
$$

This is an infinite-dimensional transfer function due to the existence of an infinite number of modes. We notice that (4) does not include any modal dampings. In reality, however, each mode is lightly damped. Therefore, a more precise version of (4) can be written as

$$
G(s)=\sum_{i=1}^{\infty} \frac{F_{i}}{s^{2}+2 \zeta_{i} s+\omega_{i}^{2}} .
$$

It is a very difficult task to determine modal structural dampings using physical principles. Therefore, $\zeta_{i}$ 's are often determined by experiments. In this paper, we ignore the effect of modal dampings for the reasons that will be explained later. However, it is straightforward to extend this work to include the effect of modal dampings.

In a typical control design scenario, the designer is often interested only in a particular bandwidth. Therefore, an approximate model of the system is needed that best represents the dynamics of the system in the prescribed frequency range. A natural choice in this case is to simply ignore the modes which correspond to the frequencies that lie outside of the bandwidth of interest. For instance, if $\omega_{N}$ is equivalent or larger than the highest frequency of interest, one may choose to approximate $G(s)$ by

$$
G_{N}(s)=\sum_{i=1}^{N} \frac{F_{i}}{s^{2}+\omega_{i}^{2}} .
$$

A drawback of this approach is that the truncated higher order modes may contribute to the low-frequency dynamics in the form of distorting zero locations. Reference [2] suggests a way of dealing with this problem. The idea that is put forward in [2] is to allow for a constant feed-through term in (5) to account for the compliance of omitted higher order modes of (4). That is, to approximate $G(s)$ by

$$
\hat{G}(s)=G_{N}(s)+K
$$

where $K=\sum_{i=N+1}^{\infty} F_{i} / \omega_{i}^{2}$. The logic behind this choice of $K$ is that at lower frequencies one can ignore the effect of dynamical responses of higher order modes since they are much smaller than the force responses at those frequencies. Although an approximation, [2] shows that $K$ is a good representation of the effect of higher order modes on $G_{N}(s)$.

This paper is an attempt to find an optimal value for $K$. In other words, we intend to determine $K$ such that the effect of higher order modes on the low-frequency dynamics is minimized in some measure. Our objective here is to choose a value for $K$ such that the following cost function is minimized:

$$
\|(G(s)-\hat{G}(s)) W(s)\|_{2}^{2}
$$

where $\|f(s)\|_{2}^{2}=1 / 2 \pi \int_{-\infty}^{\infty}|f(j \omega)|^{2} d \omega$. Here, $G(s)$ and $\hat{G}(s)$ are defined as in (4) and (6) and $W(s)$ is an ideal low-pass weighting function with its cut-off frequency $\omega_{c}$ chosen to lie within the interval $\omega_{c} \in\left(\omega_{N}, \omega_{N+1}\right)$. That is, $|W(j \omega)|=1$ for $-\omega_{c} \leq \omega \leq \omega_{c}$ and 0 elsewhere. The reason for this choice of $W$ will be explained soon. To this end, it should be clear that a $K$ chosen to minimize (7) will minimize the effect of out of bandwidth dynamics of $G(s)$ on $\hat{G}(s)$ in an $\mathrm{H}_{2}$ optimal sense. Notice that the cost function (7) conveys no information on frequencies higher than $\omega_{c}$.

It is easy to see that (7) is equivalent to

$$
\left\|\left(\sum_{i=N+1}^{\infty} \frac{F_{i}}{s^{2}+\omega_{i}^{2}}-K\right) W(s)\right\|_{2}^{2} .
$$

The fact that $W$ is chosen to be an ideal low-pass filter with its cutoff frequency lower than the first out-of-bandwidth pole of $G$, guarantees that (8) will remain finite. Let

$$
\tilde{G}(s)=\sum_{i=N+1}^{\infty} \frac{F_{i}}{s^{2}+\omega_{i}^{2}} .
$$

It is straightforward to show that (8) is equivalent to

$$
\|\tilde{G} W\|_{2}^{2}+K^{2}\|W\|_{2}^{2}-K(\langle\tilde{G} W, W\rangle+\langle W, \tilde{G} W\rangle)
$$

where $\langle f, g\rangle=(1 / 2 \pi) \int_{-\infty}^{\infty} f^{*}(j \omega) g(j \omega) d \omega$. It is straightforward to verify that the $K$ that minimizes (9) is given by

$$
K=\frac{\langle\tilde{G} W, W\rangle+\langle W, \tilde{G} W\rangle}{2\|W\|_{2}^{2}}
$$

which is equivalent to

$$
K=\frac{\int_{-\infty}^{\infty} \operatorname{Re}(\tilde{G}(j \omega))|W(j \omega)|^{2} d \omega}{\int_{-\infty}^{\infty}|W(j \omega)|^{2} d \omega}
$$

where $\operatorname{Re}(f)$ represents the real part of the complex number $f$, and consequently

$$
K=\frac{\int_{-\infty}^{\infty}\left(\sum_{i=N+1}^{\infty} \frac{F_{i}}{\omega_{i}^{2}-\omega^{2}}\right)|W(j \omega)|^{2} d \omega}{\int_{-\infty}^{\infty}|W(j \omega)|^{2} d \omega} .
$$




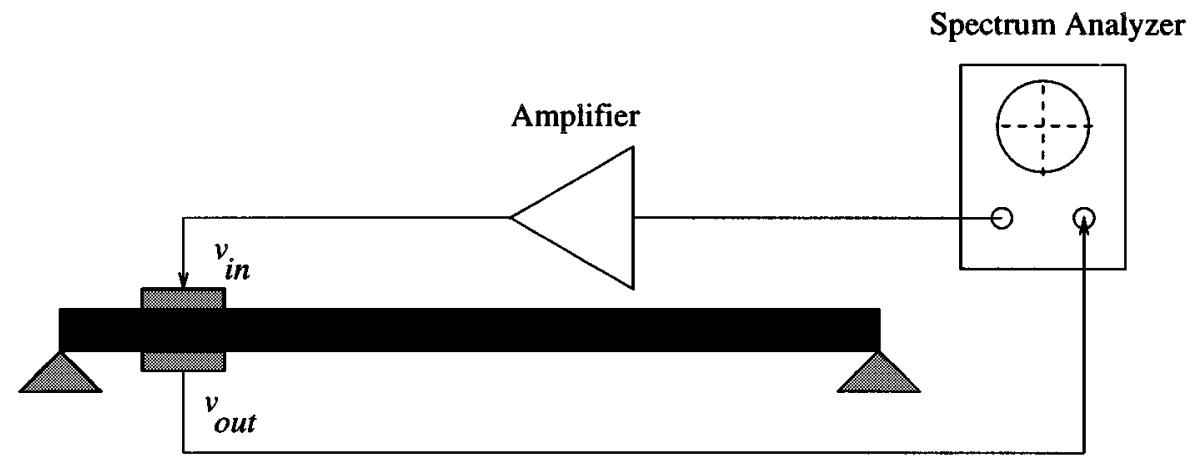

Fig. 2. The experimental setup.

TABLE I

PARAMETERS OF THE PIEZOELeCtric LAMINATE BEAM

\begin{tabular}{l|c}
\hline Beam Length, $L$ & $0.6 \mathrm{~m}$ \\
\hline Beam width & $0.05 \mathrm{~m}$ \\
\hline Beam thickness, $t_{b}$ & $0.003 \mathrm{~m}$ \\
\hline \hline Piezoceramic position, $r_{1}$ & $0.05 \mathrm{~m}$ \\
\hline Piezoceramic position, $r_{2}$ & $0.12 \mathrm{~m}$ \\
\hline Charge Constant, $d_{31}$ & $-210 \times 10^{-12} \mathrm{~m} / \mathrm{v}$ \\
\hline Voltage Constant, $g_{31}$ & $-11.5 \times 10^{-3} \mathrm{Vm} / \mathrm{N}$ \\
\hline Coupling Coefficient & 0.340 \\
\hline Capacitance, $C_{a p}$ & $104.82 \mathrm{nF}$ \\
\hline Piezoceramic width & $0.025 \mathrm{~m}$ \\
\hline Piezoceramic thickness $t_{a}$ & $0.25 \times 10^{-3} \mathrm{~m}$ \\
\hline
\end{tabular}

TABLE II

FIRST EIGHT RESONANT FREQUENCIES OF THE SIMPLY-SUPPORTED BEAM

\begin{tabular}{c|c||c|c}
\hline Mode & $f_{i}(\mathrm{~Hz})$ & Mode & $f_{i}(\mathrm{~Hz})$ \\
\hline 1 & 21.3 & 5 & 466.63 \\
\hline 2 & 77.13 & 6 & 670.59 \\
\hline 3 & 169.92 & 7 & 911.63 \\
\hline 4 & 299.74 & 8 & 1189.7 \\
\hline
\end{tabular}

Hence, to obtain the optimal $K$, one has to carry out the following integration:

$$
K=\frac{1}{2 \omega_{c}} \int_{-\omega_{c}}^{\omega_{c}} \sum_{i=N+1}^{\infty} \frac{F_{i}}{\omega_{i}^{2}-\omega^{2}} d \omega .
$$

The optimal value of $K$ is found to be

$$
K_{\mathrm{opt}}=\frac{1}{2 \omega_{c}} \sum_{i=N+1}^{\infty} \frac{F_{i}}{\omega_{i}} \ln \left(\frac{\omega_{i}+\omega_{c}}{\omega_{i}-\omega_{c}}\right) .
$$

At this point, we try to show how $K_{\text {opt }}$ is related to the value of $K$ suggested by [2]. We know that for $x>0$, the term $\ln (x)$ can be expanded as (see [15, p. 52])

$$
\ln (x)=2 \sum_{n=1}^{\infty} \frac{1}{2 n-1}\left(\frac{x-1}{x+1}\right)^{(2 n-1)} .
$$

If we use the first term of the series to approximate $\ln \left(\left(\omega_{i}+\omega_{c}\right) /\left(\omega_{i}-\omega_{c}\right)\right)$ in (14), we obtain

$$
K=\sum_{i=N+1}^{\infty} \frac{F_{i}}{\omega_{i}^{2}} .
$$

Hence, we recover the result of [2]. Therefore, the value of $K$ suggested by [2] approximates the optimal $K$ which minimizes (7).

To this end, we point out that this result can have direct implications on the development of system identification techniques for piezoelectric laminates. Indeed, if a model is to be fitted to the experimental data, care has to be taken that a proper model which consists of a sufficient number of second order resonant terms and a feed-through term is chosen.

\section{THE EXPERIMENT}

In this section we validate our model correction technique through simulations and an experiment on a piezoelectric laminate. The experiment is depicted in Fig. 2. The structure consists of a $60-\mathrm{cm}$ long uniform aluminum beam of a rectangular cross section $(50 \mathrm{~mm} \times 3 \mathrm{~mm})$. The beam is pinned at both ends. A pair of piezoelectric ceramic elements are attached symmetrically to either side of the beam, $50 \mathrm{~mm}$ away from one end of the beam. The piezoceramic elements used in our experiment are PIC151 patches. These patches are 25-mm wide, $70-\mathrm{mm}$ long, and $0.25-\mathrm{mm}$ thick. The physical parameters of PIC151 are given in Table I.

In this experiment, a Hewlett Packard model 89410 A vector analyzer is used to determine the frequency response of the piezoelectric laminate. In [3], it is argued that the transfer function of the laminate has to be modified to allow for the effect of finite input resistance of the measurement device. If the total resistance of the measuring device is $R_{m}$ and the total capacitance of the sensing piezoelectric patch is $C_{t}$, in the transfer function (3), $C_{s}$ should be replaced with

$$
C_{s}\left(\frac{s R_{m} C_{\text {ap }}}{s R_{m} C_{\text {ap }}+1}\right) \text {. }
$$

The input resistance of HP89410A is $1 \mathrm{M} \Omega$. To reduce the effect of this low input resistance on our measurement, a Tektronix P6201 active probe with $1 \mathrm{M}-\Omega$ input resistance is used. Therefore, the high-pass cutoff frequency $f_{c}=1 / R_{m} C_{\text {ap }}$ is moved below $1 \mathrm{~Hz}$. 


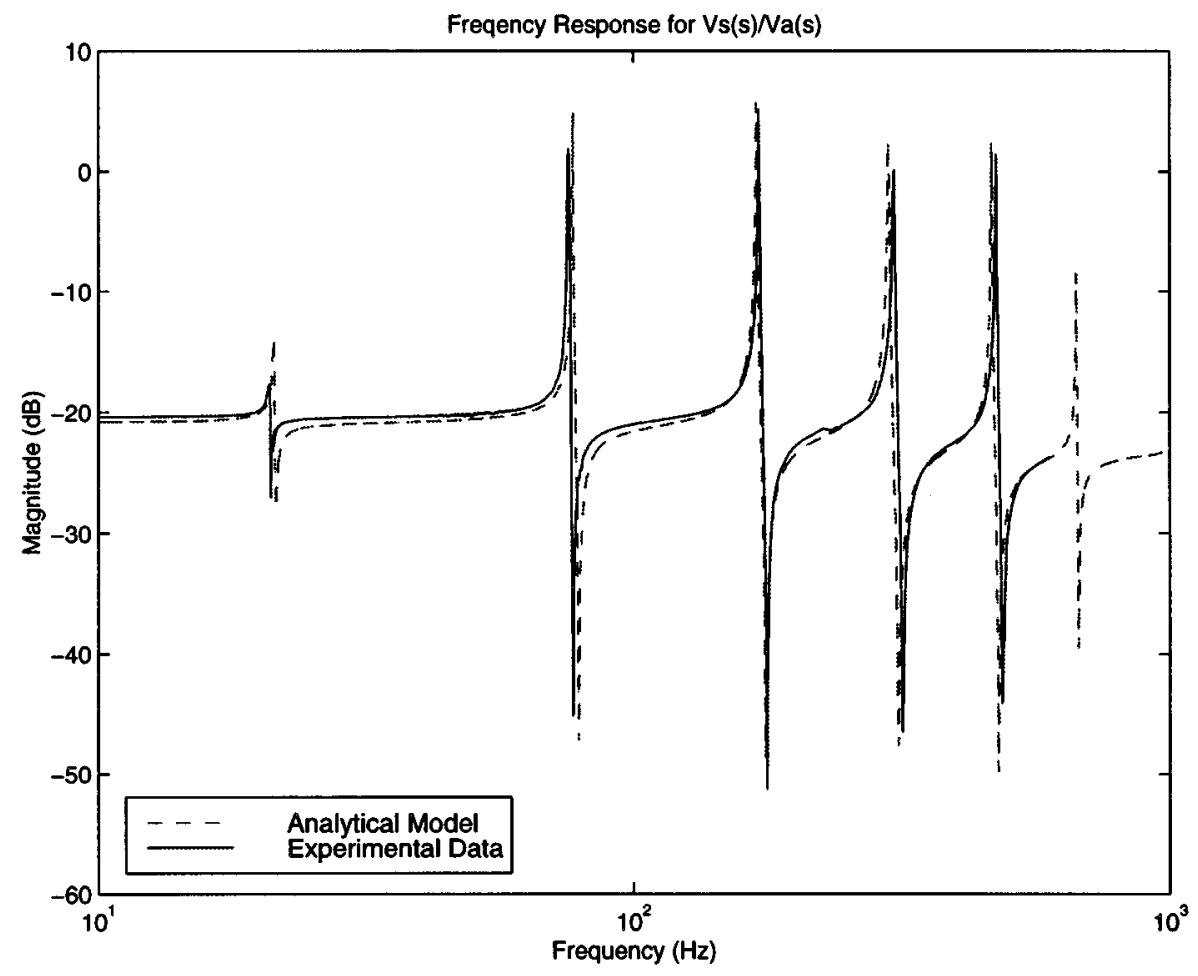

Fig. 3. Comparison of the frequency responses of the 150 mode model of the laminate with the experimental data.

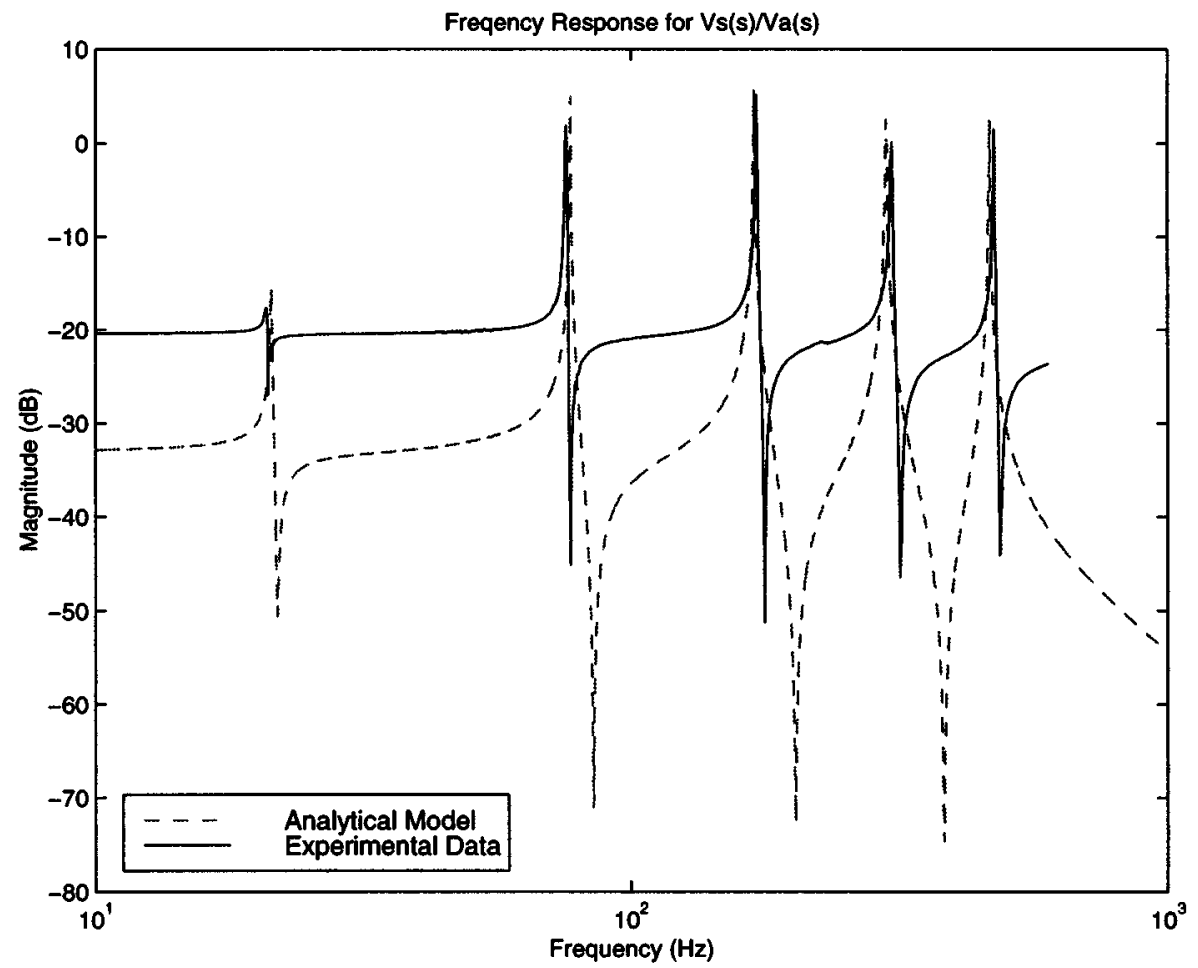

Fig. 4. Comparison of the frequency responses of the five mode model of the laminate with the measured frequency response.

A model of the beam is determined using the (3). Here, the mode shapes and resonant frequencies are determined as explained in Section II. Our model consists of the first 150 modes of the beam. This is enough to give us an accurate model of the structure in the frequency range of up to 500 $\mathrm{Hz}$. There are five modes within this particular bandwidth.
Hence, we are interested in working with a five mode model of the laminate.

In Fig. 3 we compare the frequency response of the beam based on its first 150 modes with our experimental measurements in up to $500-\mathrm{Hz}$ range. It can be observed that the two models are very close. In Fig. 4 we plot the experi- 


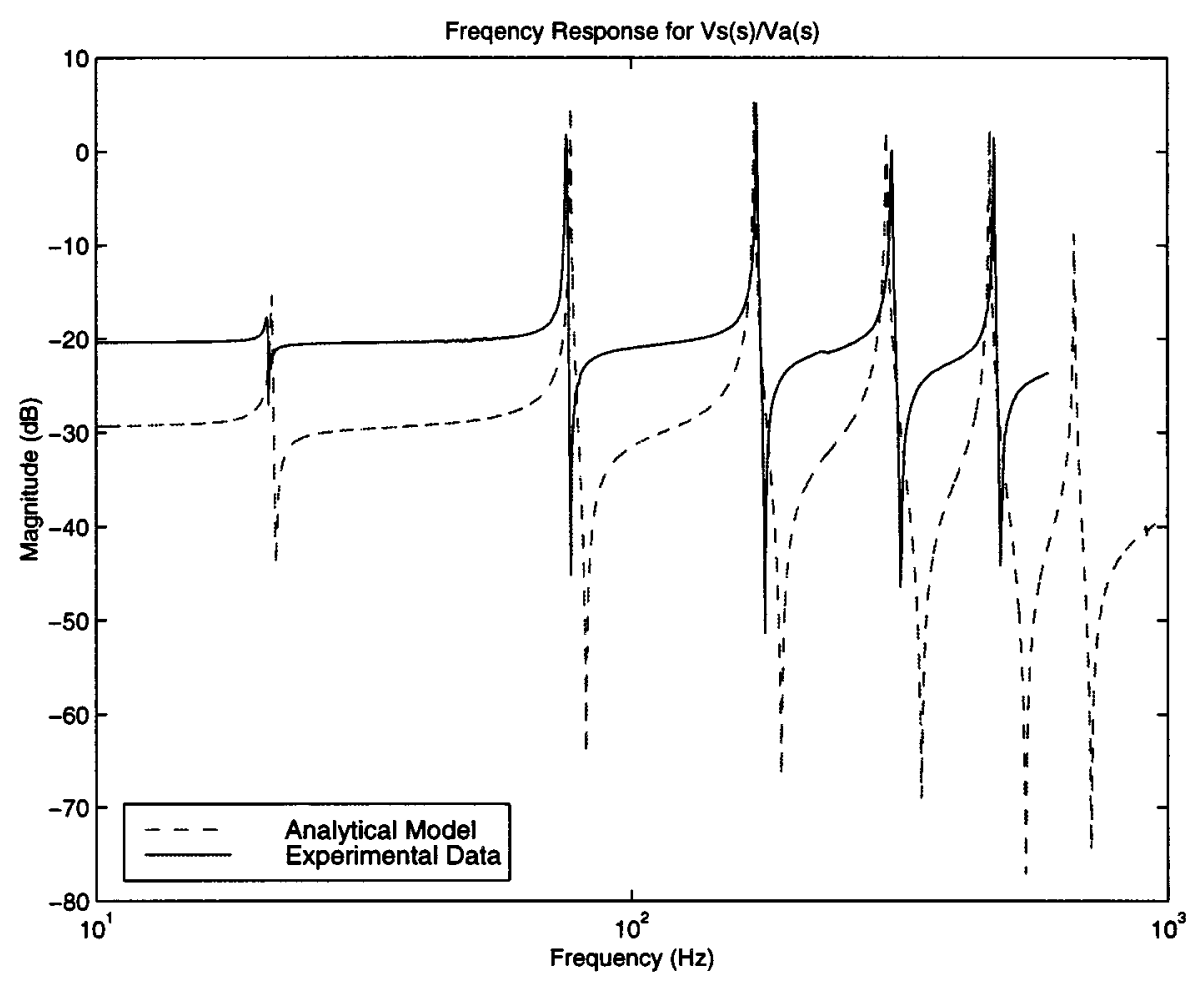

Fig. 5. Comparison of the frequency responses of the 50 mode model of the laminate with the measured frequency response.

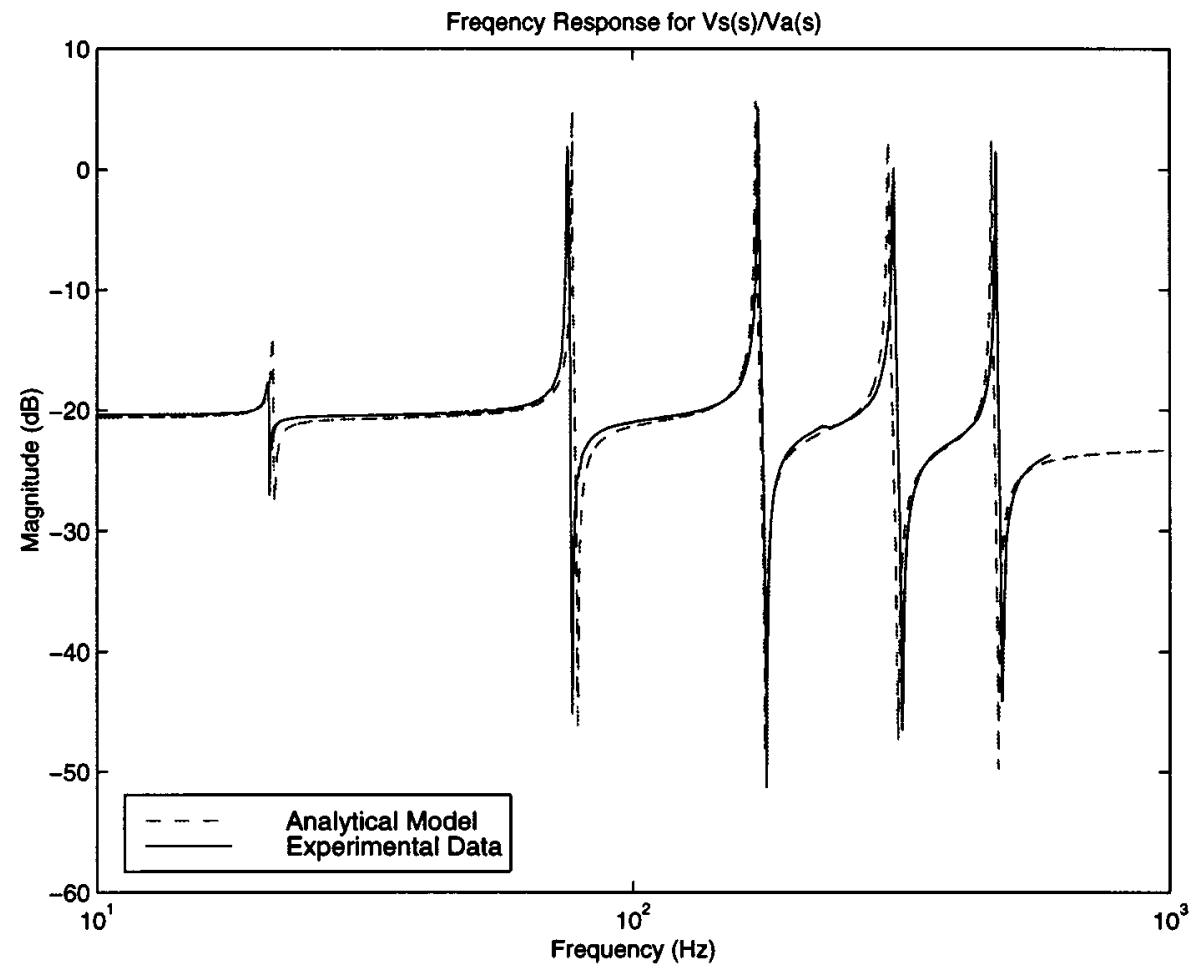

Fig. 6. Comparison of the frequency responses of the corrected model of the laminate with the measured frequency response.

mental data and the frequency response of the five mode model. This figure clearly shows the error that is introduced by truncation. In Fig. 5 we plot our experimental data and the frequency response of the 50 mode model of the beam. Some improvement can be observed, however, the difference between the frequency responses of the two systems is still unacceptably high. Now, we approximate the effect of truncated modes on the five mode model of the system via the (14). The frequency $\omega_{c}$ is chosen to be $\left(\omega_{5}+\omega_{6}\right) / 2$, i.e., $3572.7 \mathrm{rad} / \mathrm{s}$. In Fig. 6, we plot and compare the frequency response of the corrected five mode model of the beam with our experimental measurements. It can be observed that the 
difference between the two frequency responses in the frequency range of interest is minimal.

\section{CONCLUSION}

In this paper we proposed a model correction methodology that can be used to minimize the effect of truncated modes on low-frequency models of piezoelectric laminates. This is done by adding a feed-through term to the truncated model of the laminate. This term is chosen such that the $\mathrm{H}_{2}$ norm of the error system is minimized. We also presented experimental results which support our model correction technique.

\section{ACKNOWLEDGMENT}

The author wishes to thank S. Behrens for his assistance in the experimental part of this research.

\section{REFERENCES}

[1] L. Meirovitch, Elements of Vibration Analysis, 2nd ed. New York: McGraw-Hill, 1986.

[2] R. L. Clark, "Accounting for out-of-bandwidth modes in the assumed modes approach: Implications on colocated output feedback control," Trans. ASME J. Dynamic Syst., Measurement, Contr, vol. 119, pp. 390-395, 1997.

[3] H. R. Pota and T. E. Alberts, "Multivariable transfer functions for a slewing piezoelectric laminate beam," ASME J. Dynamic Syst., Measurement, Contr., vol. 117, pp. 353-359, Sept. 1995.

[4] T. E. Alberts, T. V. DuBois, and H. R. Pota, "Experimental verification of transfer functions for a slewing piezoelectric laminate beam," Contr. Eng. Practice, vol. 3, no. 2, pp. 163-170, 1995.

[5] H. R. Pota and T. E. Alberts, "Vibration analysis using symbolic computation software," presented at the Proc. 1997 Amer. Contr. Conf., Albuquerque, NM, June 1997.

[6] S. O. R. Moheimani, I. R. Petersen, and H. R. Pota, "Broadband disturbance attenuation over an entire beam," J. Sound Vibration, vol. 227, no. 4, pp. 807-832, 1999.
[7] S. O. R. Moheimani, H. R. Pota, and I. R. Petersen, "Spatial balanced model reduction for flexible structures," Automatica, vol. 35, no. 2, pp. 269-277, 1999.

[8] _ "Active vibration control—A spatial LQR approach," presented at the Proc. Contr. 97, Sydney, Australia, Oct. 20-22, 1997, pp. 622-627.

[9] _ - "Active control of noise and vibration in acoustic ducts and flexible structures-A spatial control approach," presented at the Proc. 1998 Amer. Contr. Conf., Philadelphia, PA, June 1998.

[10] S. O. R. Moheimani, I. R. Petersen, and H. R. Pota, "Broadband disturbance attenuation over an entire beam," in Proc. Europ. Contr. Conf., Brussels, Belgium, July 1-4, 1997. To appear in the J. Sound Vibration.

[11] T. E. Alberts and J. A. Colvin, "Observations on the nature of transfer functions for control of piezoelectric laminates," J. Intell. Material Syst. Structures, vol. 8, no. 5, pp. 605-611, 1991.

[12] C. R. Fuller, S. J. Elliot, and P. A. Nelson, Active Control of Vibration. New York: Academic, 1996.

[13] A. Baz and S. Poh, "Performance of an active control system with piezoelectric actuators," J. Sound Vibration, vol. 126, no. 2, pp. 327-343, 1988.

[14] A. R. Fraser and R. W. Daniel, Perturbation Techniques for Flexible Manipulators. Boston, MA: Kluwer, 1991.

[15] I. S. Gradsteyn and I. M. Ryshik, Table of Integrals, Series, and Products, 4th ed. New York: Academic, 1994.

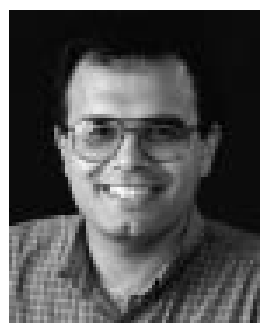

S. O. Reza Moheimani was born in Iran in 1967. He received the B.Sc. degree from Shiraz University, Iran, in 1990 and the M.Eng.Sc. and Ph.D. degrees from the University of New South Wales, Australia, in 1993 and 1996, all in electrical and electronic engineering.

In 1996 he was a Postdoctoral Research Fellow at the School of Electrical and Electronic Engineering, Australian Defence Force Academy, Canberra, Australia. In 1997, he joined the Department of Electrical and Computer Engineering at the University of Newcastle, where he is currently a Senior Lecturer. He is a member of the Centre for Integrated Dynamics and Control, which is an Australian Government Special Research Centre. His interests include robust control and filtering, active control of noise and vibrations, smart structures, and signal processing. 